\title{
SOME STEREOLOGICAL PRINCIPLES IN MORPHOMETRIC CYTOLOGY*
}

\author{
JOEL N. FRANKLIN†
}

\begin{abstract}
In this paper several statistical methods are developed that are of use in morphometric cytology, for the purpose of obtaining three-dimensional information from two-dimensional measurements made on cell sections. An estimator is described for counting $N_{V}$, the number of organelles (e.g., mitochondria) per unit of cellular volume. Unlike those currently employed, this estimator for $N_{V}$ does not depend on the sizes of the organelle bodies. The remainder of the paper discusses the axis ratios, $t$, of elliptical cross sections that are the intersections of an ellipsoid with random planes. For prolate spheroids, an explicit formula is developed for the probability density. For general ellipsoids, a numerical method is presented for calculating the distribution function. As one approach toward deriving information about organelle shape, frequency distributions of $t$ derived by these techniques may be compared with experimental frequency distributions of the axis ratios of organelle cross sections.
\end{abstract}

1. Introduction. In studying the organization and dynamics of the various organelles within the cell (for example, mitochondria), cell biologists often employ a morphometric analysis of random sections through the structures of interest. The biological interpretation of the results is greatly facilitated if three-dimensional information can be derived from the two-dimensional measurements.

One particularly important three-dimensional parameter is $N_{V}$, the number of organelle bodies per unit of cellular volume. Currently used estimators for $N_{V}$ depend on a knowledge of the sizes of the bodies. For example, the estimator of Weibel and Gomez [10] and Knight et al. [6] for spheres is

$$
\text { estimated } N_{V}=K(\pi / 6)^{1 / 2} N_{A}^{3 / 2} V_{V}^{-1 / 2}
$$

where $N_{A}$ and $V_{V}$ are found from experiment: $N_{A}$ is the number of bodies intersected per unit of cellular profile area; $V_{V}=A_{A}$ is the fraction of cellular profile area occupied by organelle profiles. But

$$
K=\left(D_{3} / D_{1}\right)^{3 / 2}
$$

where $D_{m}$ is the $m$ th moment of the size distribution. So the estimator (1.1) depends on a knowledge of the organelle size distribution. Here, in $\S \S 2-6$, we shall discuss an estimator for $N_{V}$ that is size-independent.

Organelle shape is another important type of three-dimensional information. In $\S \S 7$ and 8 , we shall discuss the axis ratios, $t$, of elliptical cross sections that are the intersections of an ellipsoid with random planes. If the ellipsoid is a prolate spheroid, we obtain an explicit formula for the probability density, $p(t)$. If the ellipsoid is general, we represent the distribution function, $P(t)$, by an integral; and we show how to calculate $P(t)$ and $p(t)$ by a numerical method. Frequency distributions of $t$ derived by these techniques may be compared with experimental

\footnotetext{
* Received by the editors June 4, 1976, and in revised form October 29, 1976.

$\dagger$ Department of Applied Mathematics, California Institute of Technology, Pasadena, California 91125 .
} 
frequency distributions of the axis ratios of organelle profiles, as an approach toward deducing information regarding the shape of the organelles within the cell.

The methods presented in this paper have been employed in a recent study of the growth and division of mitochondria in human cells. ${ }^{1}$

For a review of the use of stereological principles in cytology, see Weibel[11].

2. Spheres. Suppose there is a single sphere of radius $R$ inside the unit cube. Make a random section of area 1 inside the cube. The section may or may not cut the sphere (the probability of cutting the sphere is $2 R$ ).

Define a random variable, $w$, as follows: If the sphere is not cut, set $w=0$. If the sphere is cut, let $A$ be the area of the circular cross section, and set $w=1 / \sqrt{A}$.

The random variable $w$ has an expected value, $E(w)$. To evaluate $E(w)$, suppose $x$ is the distance between the center of the sphere and the random section. Then

$$
E(w)=\int_{-R}^{R}[A(x)]^{-1 / 2} d x
$$

where $A(x)$ is the area of a circle cut from the sphere by a plane at distance $x$ from the center of the sphere. Then

$$
A(x)=\pi\left(R^{2}-x^{2}\right)
$$

and (2.1) gives

$$
\begin{gathered}
E(w)=\int_{-R}^{R}\left[\pi\left(R^{2}-x^{2}\right)\right]^{-1 / 2} d x, \\
E(w)=\sqrt{\pi} .
\end{gathered}
$$

Note that $E(w)$ is independent of the size of the sphere. This enables us to estimate the number of spheres in a unit volume by random sectioning.

Suppose there are $N$ spheres in the unit cube. Let their radii be $R_{1}, R_{2}$, $\cdots, R_{N}$. Take a random section of unit cellular area. Define the random variable $w_{k}$ to be the variable $w$ for the $k$ th sphere: $w_{k}=0$ if the $k$ th sphere is not cut; $w_{k}=1 / \sqrt{A_{k}}$ if the $k$ th sphere is cut. Then (2.4) implies $E\left(w_{k}\right)=\sqrt{\pi}$, and

$$
E\left(w_{1}+w_{2}+\cdots+w_{N}\right)=N \sqrt{\pi} \text {. }
$$

How can we use this formula? Suppose we have $N$ spheres in the unit cube; the number $N$ is unknown, and we want to estimate it. We take a random section of unit area. This cuts out some circular cross sections (or it may cut out no cross sections if the random slice misses all the spheres). Suppose $M$ circular areas are cut out: $B_{1}>0, \cdots, B_{M}>0$.

The observed areas $B_{1}, \cdots, B_{m}$ are just the positive numbers in the set $A_{1}, \cdots, A_{N}$; in other words, each $A_{i}$ is one of the $B_{j}$ if and only if $A_{i} \neq 0$. Therefore, in the notation of (2.5),

$$
w_{1}+\cdots+w_{N}= \begin{cases}B_{1}^{-1 / 2}+\cdots+B_{M}^{-1 / 2} & \text { if } M \geqq 1, \\ 0 & \text { if } M=0 .\end{cases}
$$

${ }^{1}$ J. W. Posakony, J. M. England and G. Attardi, Mitochondrial growth and division during the cell cycle in HeLa cells, submitted for publication. 
Therefore, we may estimate $N$ by

$$
\pi^{-1 / 2}\left(B_{1}^{-1 / 2}+\cdots+B_{M}^{-1 / 2}\right)
$$

if $M \geqq 1$ or by 0 if $M=0$. The expected value of this sum equals

$$
\pi^{-1 / 2} E\left(w_{1}+\cdots+w_{N}\right)=N \text {. }
$$

3. Convex bodies of the same shape. We can generalize these results as follows. Suppose $K$ is a convex body with diameter $\delta \ll 1$. First place $K$ inside the unit cube in any way so that the distance of $K$ from all faces of the cube is greater than $\delta$. Then fix a point $P_{0}$ in $K$, and rotate $K$ about $P_{0}$ at random uniformly with respect to Haar or kinematic measure over the rotation group. This random rotation fixes the orientation of $K$.

Now take a random unit-square slice inside the cube parallel to a face. Define a random variable, $w$, as follows: If the body is not cut, set $w=0$. If the body is cut, let $A$ be the area of the cross section, and set $w=1 / \sqrt{A}$. (At most one region can be cut out, since the body is convex.)

The random variable $w$ has an expected value, $E(w)$, which we can evaluate as follows: Regard the point $P_{0}$ in $K$ as the origin of a coordinate system. Let $\mathbf{u}$ be a unit vector. Define the support-function

$$
H(\mathbf{u})=\max \mathbf{p} \cdot \mathbf{u}
$$

for all $\mathbf{p}$ in the convex body $K$. The positive number $H(\mathbf{u})$ is the length of the orthogonal projection of the body $K$ on the half-line originating at $P_{0}$ and extending in the direction $\mathbf{u}$. It is also the distance from $P_{0}$ to the plane tangent to $K$ whose outer normal is $\mathbf{u}$.

Let $P l$ be any plane with normal $\mathbf{u}$. Let $x$ equal the constant value of the inner product $\mathbf{p} \cdot \mathbf{u}$ for all points $\mathbf{p}$ on the plane $P l$. Thüs, $x$ may be positive or negative; $x=0$ in the plane $P l$ passes through the origin, $P_{0}$. The plane cuts the body $K$ in some positive area if and only if

$$
-H(-\mathbf{u})<x<H(\mathbf{u})
$$

Let $A(x, \mathbf{u})$ be the value of the area cut out.

For example, if $K$ is the sphere of radius $R$, and if $P_{0}$ is the center of the sphere, then $H(\mathbf{u})=R$ for all $\mathbf{u}$. Then (3.2) has the form $-R<x<R$, and $A(x$, $\mathbf{u})=\pi\left(R^{2}-x^{2}\right)$.

In general, the expected value of $w$ is the average over all directions, $\mathbf{u}$, of the integral

$$
I(\mathbf{u})=\int_{-H(-\mathbf{u})}^{H(\mathbf{u})}[A(x, \mathbf{u})]^{-1 / 2} d x .
$$

Thus, if $d S$ is the differential of area on the unit sphere $|\mathbf{u}|=1$, the expectation $E(w)$ is the average

$$
J=\frac{1}{4 \pi} \int_{|\mathbf{u}|=1} I(\mathbf{u}) d S
$$


The number $J$ depends only on the body $K$. It is unaffected by rigid motions of $K$. It is even unaffected by scalings of $K$ : if $K^{\prime}$ is any body geometrically similar to $K$, then $J\left(K^{\prime}\right)=J(K)$. (For instance: $J=\sqrt{\pi}$ for spheres of all sizes.) Since $J$ depends only on the shape of $K$ and not on its size, we will call $J$ a shape constant of $K$. (It is easy to define other shape constants by replacing the integrand in (3.3) by some other functional of the cross section that has dimension $1 /$ length; for instance, one could use the perimeter of the cross section divided by the area.)

Suppose there are $N$ convex bodies in the unit cube. Suppose they are all geometrically similar, though they may have different sizes. We want to estimate $N$.

We take a random slice of unit area in the cube. We observe $M$ cross sections cut from the bodies (so $M$ satisfies $0 \leqq M \leqq N$ ). As in (2.6), we form the statistic

$$
N^{*}= \begin{cases}J^{-1}\left(B_{1}^{-1 / 2}+\cdots+B_{M}^{-1 / 2}\right) & \text { if } M \geqq 1, \\ 0 & \text { if } M=0,\end{cases}
$$

where $J$ is the common shape constant of the $N$ bodies, and where $B_{1}, \cdots, B_{M}$ are the areas of the observed cross sections. The statistic $N^{*}$ is an unbiased estimator of $N$; as in (2.8), we find

$$
E N^{*}=J^{-1} E\left(w_{1}+\cdots+w_{N}\right)=N
$$

A note of caution: One can use this technique only for a convex body with continuous normal on the boundary. Otherwise, the shape constant $J$ may be infinite. For instance, $J=\infty$ for all polyhedra (tetrahedron, cube, etc.).

Even for smooth bodies with finite $J$, the random variable $N^{*}$ defined by (3.5) has infinite variance. Even so, $N^{*}$ is a usable estimate: given any $\varepsilon>0$, if $N_{1}^{*}, \cdots, N_{n}^{*}$ are independent samples of $N^{*}$, the probability that

$$
\left|\frac{1}{n}\left(N_{1}^{*}+\cdots+N_{n}^{*}\right)-N\right|>\varepsilon
$$

tends to zero as $n$ tends to $\infty$. That follows from Khintchine's form of the law of large numbers, which does not require a finite variance; see the exposition by W. Feller [1, pp. 243-248].

4. Convex bodies of different shapes. Suppose there are $N$ smooth convex bodies in the unit cube. Suppose they have shape constants $J_{1}, \cdots, J_{N}$. (If the bodies are geometrically similar, the $J$ 's are equal.) We want to estimate $N$.

We take a random slice of unit area. The slice cuts out $M$ cross sections $(0 \leqq M \leqq N)$. If $M \geqq 1$, let $B_{1}, \cdots, B_{M}$ be the areas cut out. Then form the estimator

$$
N^{*}= \begin{cases}J^{-1}\left(B_{1}^{-1 / 2}+\cdots+B_{M}^{-1 / 2}\right) & \text { if } M \geqq 1, \\ 0 & \text { if } M=0,\end{cases}
$$

where $J$ is the mean shape constant

$$
J=N^{-1}\left(J_{1}+\cdots+J_{N}\right)
$$


AsSERTION. $N^{*}$ is an unbiased estimator of $N$ :

$$
E N^{*}=N \text {. }
$$

Proof. As before, let $w_{i}$ be the random variable equal to 0 if the body $K_{i}$ is not cut, or $w_{i}=A_{i}^{-1 / 2}$ if $K_{i}$ is cut, where $A_{i}$ is the area of intersection. By definition, the shape constant $J_{i}$ equals the expected value $E w_{i}$. But

$$
\sum_{i=1}^{N} w_{i}= \begin{cases}\sum_{A_{i}>0} A_{i}^{-1 / 2}=B_{1}^{-1 / 2}+\cdots+B_{M}^{-1 / 2} & \text { if } M \geqq 1, \\ 0 & \text { if } M=0 .\end{cases}
$$

By (4.1), $\sum w_{i}=J N^{*}$. If we take expected values, we find

$$
\sum_{i=1}^{N} J_{1}=J \cdot E\left(N^{*}\right)
$$

Now $\sum J_{i}=N J$ by (4.2); and the assertion (4.3) follows.

5. Ellipsoids. Now we will evaluate the shape-constant $J$ for the general ellipsoid. We shall use the notation of $\S 3$.

Let the ellipsoid have the semi-axes $a_{1} \leqq a_{2} \leqq a_{3}$. Since $J$ is independent of scaling, it depends only on two of the axis-ratios, say $a_{2} / a_{1}$ and $a_{3} / a_{1}$.

Suppose that the coordinate axes $x_{1}, x_{2}, x_{3}$ are taken in the directions of the principal axes, and that the origin $P_{0}$ is taken at the center of the ellipsoid. Then the surface of the ellipsoid has the equation.

$$
x_{1}^{2} / a_{1}^{2}+x_{2}^{2} / a_{2}^{2}+x_{3}^{2} / a_{3}^{2}=1
$$

Let $\mathbf{u}=\left(u_{1}, u_{2}, u_{3}\right)$ be a unit vector. Then

$$
H(\mathbf{u})=\max \left(x_{1} u_{1}+x_{2} u_{2}+x_{3} u_{3}\right)
$$

for all points $\mathbf{x}$ in the ellipsoid. It is easy to see that the maximum is attained for $\mathbf{x}$ on the surface (5.1) (and not for $\mathbf{x}$ in the interior). Now calculus gives

$$
H(\mathbf{u})=\left(a_{1}^{2} u_{1}^{2}+a_{2}^{2} u_{2}^{2}+a_{3}^{2} u_{3}^{2}\right)^{1 / 2} .
$$

Now to compute $A(x, \mathbf{u})$ : Parallel planes cut an ellipsoid in similar ellipses. When $x=0, A(0, \mathbf{u})$ is the area of the cross section through the origin with normal u. When $x=H(\mathbf{u}), A(H(\mathbf{u}), \mathbf{u})=0$, because $H(\mathbf{u})$ is the distance to the tangent plane with normal $\mathbf{u}$. For $-H(\mathbf{u}) \leqq x \leqq H(\mathbf{u})$ analytic geometry yields

$$
A(x, \mathbf{u})=A(0, \mathbf{u})\left[1-x^{2} / H^{2}(\mathbf{u})\right] .
$$

For example, if the ellipsoid is a sphere of radius $R$,

$$
A(x, y)=\pi R^{2}\left[1-x^{2} / R^{2}\right]=\pi\left(R^{2}-x^{2}\right) .
$$

Integrating from $-H(\mathbf{u})$ to $H(\mathbf{u})$, we find

$$
\int_{-H(\mathbf{u})}^{H(\mathbf{u})} A(x, \mathbf{u}) d x=A(0, \mathbf{u}) \cdot \frac{4}{3} H(\mathbf{u}) .
$$


But this integral is just the volume of the ellipsoid, which we know equals $(4 \pi / 3) a_{1} a_{2} a_{3}$. Therefore, $A(0, \boldsymbol{u})=\pi a_{1} a_{2} a_{3} H^{-1}(\mathbf{u})$, and

$$
A(x, \mathbf{u})=\pi a_{1} a_{2} a_{3} H^{-1}(\mathbf{u})\left[1-x^{2} / H^{2}(\mathbf{u})\right] .
$$

The integral $I(\mathbf{u})$, defined in (3.3), equals

$$
\int_{-H}^{H} A^{-1 / 2} d x=\int_{-H}^{H}\left(\pi a_{1} a_{2} a_{3}\right)^{-1 / 2} H^{1 / 2}\left(1-x^{2} / H^{2}\right)^{-1 / 2} d x
$$

where $H=H(\mathbf{u})$. But

$$
\int_{-H}^{H}\left(1-x^{2} / H^{2}\right)^{-1 / 2} d x=\pi H
$$

Now (5.2') and (5.5) give

$$
I(\mathbf{u})=\pi^{1 / 2}\left(a_{1} a_{2} a_{3}\right)^{-1 / 2}\left(a_{1}^{2} u_{1}^{2}+a_{2}^{2} u_{2}^{2}+a_{3}^{2} u_{3}^{2}\right)^{3 / 4} .
$$

The shape-constant $J$ is the average over the unit sphere, $|\mathbf{u}|=1$, of the dimensionless quantity $I(\mathbf{u})$ :

$$
J=\text { Average } I(\mathbf{u})
$$

In (5.6) we insert

$$
u_{1}=(\cos \varphi) \sin \theta, \quad u_{2}=(\sin \varphi) \sin \theta, \quad u_{3}=\cos \theta
$$

Then, since $I(\mathbf{u})$ depends only on the squares of the $u_{i}$, the average (5.7) can be evaluated in the first octant:

$$
J=\frac{2}{\pi} \int_{0}^{\pi / 2} \int_{0}^{\pi / 2} I(\mathbf{u}) \sin \theta d \theta d \varphi
$$

Though we cannot evaluate the integral $J$ analytically, we can evaluate it numerically. We can use a two-dimensional trapezoidal rule, Simpson's rule, or a Monte Carlo method-see Hammersley and Handscomb [3].

6. Inequalities for the shape-constant. Sometimes we need only an approximate value for the shape-constant $J$ defined by the integral (5.8). Then we can use these inequalities:

$$
\begin{aligned}
& J \leqq \pi^{1 / 2}\left(a_{1} a_{2} a_{3}\right)^{-1 / 2}\left[\frac{1}{3}\left(a_{1}^{2}+a_{2}^{2}+a_{3}^{2}\right)\right]^{3 / 4}, \\
& J \geqq \pi^{1 / 2}\left(a_{1} a_{2} a_{3}\right)^{-1 / 2} \frac{1}{3}\left(a_{1}^{3 / 2}+a_{2}^{3 / 2}+a_{3}^{3 / 2}\right), \\
& J \geqq \pi^{1 / 2} .
\end{aligned}
$$

We will prove these inequalities for all ellipsoids. I conjecture that the last inequality, $J \geqq \sqrt{\pi}$, holds for all convex bodies.

Proof of (6.1). Hölder's inequality implies

$$
\int_{|\mathbf{u}|=1}\left(\sum a_{i}^{2} u_{i}^{2}\right)^{3 / 4} d S \leqq\left(\int \sum a_{i}^{2} u_{i}^{2} d S\right)^{3 / 4}\left(\int d S\right)^{1 / 4} .
$$


But $\int d S=4 \pi$, and $\int u_{i}^{2} d S=4 \pi / 3$. Therefore,

$$
\int\left(\sum a_{i}^{2} u_{i}^{2}\right)^{3 / 4} d S \leqq\left(\frac{4 \pi}{3} \sum a_{i}^{2}\right)^{3 / 4}(4 \pi)^{1 / 4} .
$$

But (5.6) and (5.7) state

$$
J=\pi^{1 / 2}\left(a_{1} a_{2} a_{3}\right)^{-1 / 2}(4 \pi)^{-1} \int_{|\mathbf{u}|=1}\left(\sum a_{i}^{2} u_{i}^{2}\right)^{3 / 4} d S .
$$

Now (6.5) yields the upper bound (6.1).

Proof of (6.2) and (6.3). Since $t^{3 / 4}$ is a concave function of $t$, the $\frac{3}{4}$-power of a mean value is $\geqq$ the mean of the $\frac{3}{4}$-power:

$$
\left(\sum a_{i}^{2} u_{i}^{2}\right)^{3 / 4} \geqq \sum a_{i}^{3 / 2} u_{i}^{2}
$$

if $\sum u_{i}^{2}=1$. Integrate this inequality over the sphere $|\mathbf{u}|=1$ :

$$
\int\left(\sum a_{i}^{2} u_{i}^{2}\right)^{3 / 4} d S \geqq \frac{4 \pi}{3} \sum a_{i}^{3 / 2} .
$$

Now the definition of $J,(6.6)$, yields (6.2).

Now we apply the inequality of the arithmetic and geometric means:

$$
\frac{1}{3}\left(A_{1}+A_{2}+A_{3}\right) \geqq\left(A_{1} A_{2} A_{3}\right)^{1 / 3} .
$$

Set $A_{i}=a_{i}^{3 / 2}$. Then (6.2) implies $J \geqq \sqrt{\pi}$. For a general reference on inequalities, see Hardy, Littlewood and Pólya [4].

7. Axis-ratios for prolate spheroids. A prolate spheroid is an ellipsoid of revolution with one long axis and two equal shorter axes. Suppose a prolate spheroid is cut by a random plane. An ellipse is cut out; it has some axis-ratio $t \geqq 1$. We will find the probability density $p(t)$ for the random variable $t$.

If the coordinates $x_{1}, x_{2}, x_{3}$ are chosen along the principal axes, the spheroid has an equation

$$
x_{1}^{2} / a_{1}^{2}+x_{2}^{2} / a_{2}^{2}+x_{3}^{2} / a_{3}^{2}=1
$$

with $a_{1}=a_{2}<a_{3}$. Let $T=a_{3} / a_{1}$.

Then $1 \leqq t \leqq T$. If a section is cut normal to the $x_{3}$-axis, it is a circle and $t=1$; if it's cut parallel to the $x_{3}$-axis, $t=T$.

Let $\mathbf{u}$ be normal to the random section. Let $\theta$ be the angle between $\mathbf{u}$ and the $x_{3}$-axis. (So $t=1$ if $\theta=0$, and $t=T$ if $\theta=\pi / 2$.)

In the notation of (5.2), if $a_{1}=a_{2}=1$ and $a_{3}=T$,

$$
H(\mathbf{u})=\left(\sin ^{2} \theta+T^{2} \cos ^{2} \theta\right)^{1 / 2} .
$$

This is the half-width of the spheroid in the direction $\mathbf{u}$. The probability of cutting a section normal to $\mathbf{u}$ is directly proportional to $H(\mathbf{u})$.

The axis-ratio, $t$, of the elliptical section is a function of the angle $\theta$. Analytic geometry gives

$$
t(\theta)=T\left(\sin ^{2} \theta+T^{2} \cos ^{2} \theta\right)^{-1 / 2}
$$


So the random variable $t$ is a function of the random variable $\theta$, and we can find the probability density $p(t)$ if we can find the corresponding density $q(\theta)$.

Let a random plane have unit normal $\mathbf{u}$. Then $\mathbf{u}$ comes from a uniform distribution on the unit sphere; and the polar angle $\theta$ on the unit sphere lies in the interval $d \theta$ with probability $\frac{1}{2} \sin \theta d \theta$. Now suppose we know that the plane has normal $\mathbf{u}$; then the plane cuts the spheroid with conditional probability proportional to the half-width $H(\mathbf{u})$. So the density $q(\theta)$ is proportional to $\sin \theta$ times $H(\mathbf{u})$ :

$$
q(\theta)=c(\sin \theta) H(\mathbf{u})
$$

From (7.2), we find

$$
q(\theta)=c(\sin \theta)\left(\sin ^{2} \theta+T^{2} \cos ^{2} \theta\right)^{1 / 2} .
$$

We find the constant $c$ by setting $\int q(\theta) d \theta=1$. Then, if we set $z=\cos \theta$ for $0 \leqq \theta \leqq \pi / 2$, we get

$$
c=\left[2 \int_{0}^{1}\left(1+\left[T^{2}-1\right] z^{2}\right)^{1 / 2} d z\right]^{-1} .
$$

Now we can get $p(t)$. As $\theta$ increases from 0 to $\pi / 2$, $t$ decreases from $T$ to 1 ; as $\theta$ increases from $\pi / 2$ to $\pi, t$ increases from 1 to $T$-namely, $t(\theta)=t(\pi-\theta)$. Therefore,

$$
p(t) d t=-2 q(\theta) d \theta \quad \text { for } 0 \leqq \theta \leqq \pi / 2
$$

(the factor 2 appears because $t$ corresponds to $\theta$ and to $\pi-\theta$ ).

We compute from (7.3):

$$
d t / d \theta=-T\left(\sin ^{2} \theta+T^{2} \cos ^{2} \theta\right)^{-3 / 2}\left(T^{2}-1\right)(\sin \theta) \cos \theta .
$$

Now (7.4) and (7.6) give

$$
p(t) d t / d \theta=-2 c(\sin \theta)\left(\sin ^{2} \theta+T^{2} \cos ^{2} \theta\right)^{1 / 2}
$$

and so

$$
p(t)=2 c\left[T\left(T^{2}-1\right) \cos \theta\right]^{-1}\left(\sin ^{2} \theta+T^{2} \cos ^{2} \theta\right)^{2} .
$$

To get this as a function of $t$, we have to use (7.3). Algebraic substitution gives the final form:

$$
p(t)=k \cdot t^{-3}\left(T^{2}-t^{2}\right)^{-1 / 2} \quad(1 \leqq t \leqq T)
$$

where $k$ is the constant

$$
k=\left[\int_{1}^{T} t^{-3}\left(T^{2}-t^{2}\right)^{-1 / 2} d t\right]^{-1}
$$

8. Axis-ratios for general ellipsoids. Suppose we have an ellipsoid with three different axes. We cut it with a random plane. An ellipse is cut out, and it has some axis-ratio $t \geqq 1$. What is the probability density of the random variable $t$ ?

For prolate spheroids, the density $p(t)$ appears in (7.7). For general ellipsoids we do not have an explicit formula for $p(t)$, but we will get an integral representa- 
tion for the distribution function $P(t)$. Then we will derive a numerical method for calculating $P(t)$ and its derivative, $p(t)$.

Suppose the ellipsoid has the equation

$$
\lambda_{1} x_{1}^{2}+\lambda_{2} x_{2}^{2}+\lambda_{3} x_{3}^{2}=1
$$

where $\lambda_{1} \geqq \lambda_{2} \geqq \lambda_{3}>0$. (The semi-axes are $a_{i}=1 / \sqrt{\lambda_{i}}$ ). Let $\mathbf{u}$ be some unit vector. Cut the ellipsoid by a plane through the origin with normal $\mathbf{u}$. An ellipse is cut out. It has some equation

$$
\mu_{1} y_{1}^{2}+\mu_{2} y_{2}^{2}=1
$$

where $y_{1}$ and $y_{2}$ are the coordinates taken in the directions of the axes of the ellipse. If $\mu_{1} \geqq \mu_{2}$, the axis-ratio $t$ equals $\left(\mu_{1} / \mu_{2}\right)^{1 / 2}$, and all parallel elliptical sections have the same axis-ratio.

The numbers $\mu_{1}$ and $\mu_{2}$ are functions of $\mathbf{u}$. If $\mathbf{u}$ lies along the $x_{1}$-axis, then $\mu_{1}=\lambda_{2}, \mu_{2}=\lambda_{3}$; if $\mathbf{u}$ lies along the $x_{2}$-axis, then $\mu_{1}=\lambda_{1}, \mu_{2}=\lambda_{3}$. In general, $\mu_{1}$ and $\mu_{2}$ can be found by solving these equations of analytic geometry:

$$
\begin{aligned}
& \mu_{1}+\mu_{2}=u_{1}^{2}\left(\lambda_{2}+\lambda_{3}\right)+u_{2}^{2}\left(\lambda_{3}+\lambda_{1}\right)+u_{3}^{2}\left(\lambda_{1}+\lambda_{2}\right), \\
& \mu_{1} \mu_{2} \mu=u_{1}^{2} \lambda_{2} \lambda_{3}+u_{2}^{2} \lambda_{3} \lambda_{1}+u_{3}^{2} \lambda_{1} \lambda_{2} .
\end{aligned}
$$

A formula for $\boldsymbol{P}(\boldsymbol{a})$. The random variable $t$ lies in the interval $1 \leqq t \leqq T=$ $\left(\lambda_{1} / \lambda_{3}\right)^{1 / 2}$. If $t$ has probability density $p(t)$, it has distribution function

$$
P(t) \equiv \int_{1}^{t} p(s) d s \quad(1 \leqq t \leqq T)
$$

$P(a)$ is the probability that $t \leqq a$; for example,

$$
P(1)=\operatorname{Pr}(t \leqq 1)=0, \quad P(T)=\operatorname{Pr}(t \leqq T)=1 .
$$

We could find $t=\left(\mu_{1} / \mu_{2}\right)^{1 / 2}$ by solving the quadratic system (8.3), but it is easier to introduce the variable

$$
z \equiv t+t^{-1}=\left(\mu_{1}+\mu_{2}\right)\left(\mu_{1} \mu_{2}\right)^{-1 / 2} .
$$

The function $z(t)$ increases strictly as $t$ increases from 1 . Therefore, if $1 \leqq a \leqq T$,

$$
P(a)=\operatorname{Pr}(t \leqq a)=\operatorname{Pr}\left(z \leqq a+a^{-1}\right) .
$$

By (8.5) and (8.3), $z \leqq a+a^{-1}$ when $\mathbf{u}$ satisfies

or

$$
\mu_{1}+\mu_{2} \leqq\left(a+a^{-1}\right)\left(\mu_{1} \mu_{2}\right)^{1 / 2}
$$

$$
\begin{gathered}
u_{1}^{2}\left(\lambda_{2}+\lambda_{3}\right)+u_{2}^{2}\left(\lambda_{3}+\lambda_{1}\right)+u_{3}^{2}\left(\lambda_{1}+\lambda_{2}\right) \\
\leqq \\
\left(a+a^{-1}\right)\left(u_{1}^{2} \lambda_{2} \lambda_{3}+u_{2}^{2} \lambda_{3} \lambda_{1}+u_{3}^{2} \lambda_{1} \lambda_{2}\right)^{1 / 2} .
\end{gathered}
$$

The inequality (8.7) defines a set $U(a)$ on the unit sphere $|\mathbf{u}|=1$. We have just shown: $t \leqq a$ if and only if $\mathbf{u}$ lies in $U(a)$. So

$$
P(a)=\operatorname{Pr}(\mathbf{u} \in U(a)) .
$$


This relation will yield a formula for $P(a)$.

The probability that our ellipsoid will be cut by a plane with normal $\mathbf{u}$ is proportional to the width of the ellipsoid in the direction $\mathbf{u}$. As we have seen before, this width is twice the support function $H(\mathbf{u})$, which is given by (5.2):

$$
H(\mathbf{u})=\left(\lambda_{1}^{-1} u_{1}^{2}+\lambda_{2}^{-1} u_{2}^{2}+\lambda_{3}^{-1} u_{3}^{2}\right)^{1 / 2} .
$$

Now the probability that $\mathbf{u}$ lies in the set $U(a)$ is

$$
P(a)=C^{-1} \int_{\mathbf{u} \in U(a)} H(\mathbf{u}) d S .
$$

The constant $C$ must be chosen to make $P(a)=1$ when $U(a)$ is the whole unit sphere; therefore,

$$
C=\int_{|\mathbf{u}|=1} H(\mathbf{u}) d S \text {. }
$$

Numerical calculation of $\boldsymbol{P}$. We have found the formula $(8.10)$ for $P(a)$. This defines $P(a)$ as a constant times the integral of $H(\mathbf{u})$ over part of the unit sphere-namely, the set $U(a)$ of points u satisfying the inequality (8.7).

It is natural to calculate this kind of integral by a Monte Carlo method; see Hammersley and Handscomb [3]. Think of the unit sphere as the surface of the earth, and think of the set $U(a)$ as a country-Uruguay, for instance. Now think of $H(\mathbf{u})$ as some kind of surface density - say the number of humans per square mile. Then the integral

$$
\int_{\mathbf{u} \in U(a)} H(\mathbf{u}) d S
$$

counts the number of humans in Uruguay, and

$$
C=\int_{|\mathbf{u}|=1} H(\mathbf{u}) d S
$$

counts all the humans on earth. Then the quotient $P(a)$ in (8.10) represents the fraction of humans living in Uruguay.

To evaluate $P(a)$, we create a steady rainfall, uniform on the face of the earth. Many raindrops fall: $\mathbf{u}_{1}, \mathbf{u}_{2}, \mathbf{u}_{3}, \cdots, \mathbf{u}_{M}$. Some of these drops fall on Uruguay, some elsewhere. Look at the sum

$$
\sum_{i=1, \cdots, M} H\left(\mathbf{u}_{i}\right)
$$

Part of this sum comes from rain in Uruguay:

$$
\sum_{\mathbf{u}_{i} \in U(a) ; i=1, \cdots, M} H\left(\mathbf{u}_{i}\right) .
$$

Now the probability, $P(a)$, that a human lives in Uruguay equals the partial sum (8.15) divided by the total sum (8.14). 
We can use a computer to simulate random raindrops, $\mathbf{u}$. We need a pseudo-random $\mathbf{u}=\left(u_{1}, u_{2}, u_{3}\right)$ that belongs to the uniform distribution on the surface $|\mathbf{u}|=1$.

The set $U(a)$ is defined by the inequality (8.7), which depends only on the squares of the three components of $\mathbf{u}$; and $H(\mathbf{u})$ is defined by (8.9), which also depends only on the squares of the components. Therefore, we may use just the first octant of the unit sphere; we will generate random points $\mathbf{u}$ on the surface of the first octant.

Most scientific computing centers provide a subroutine for generating pseudo-random numbers; these are numbers $x$ that simulate samples from the uniform distribution on the interval $0<x \leqq 1$. Take three independent samples: $x_{1}, x_{2}, x_{3}$. These numbers may be regarded as the coordinates of a random point $\mathbf{x}$ inside the unit cube in the first octant.

If $x_{1}^{2}+x_{2}^{2}+x_{3}^{2} \leqq 1$, then $\mathbf{x}$ also lies inside the unit sphere $|\mathbf{x}| \leqq 1$. If $\mathbf{x}$ passes this test, we accept it; if $|\mathbf{x}|>1$, we reject it and generate a new $\mathbf{x}=\left(x_{1}, x_{2}, x_{3}\right)$. We keep this up until we get an $\mathbf{x}$ that passes the test $x_{1}^{2}+x_{2}^{2}+x_{3}^{2} \leqq 1$. (The probability that $\mathbf{x}$ passes is $\pi / 6$.)

We now have an $\mathbf{x}$ that is a random sample from the uniform distribution inside the unit sphere in the positive octant:

$$
x_{1}^{2}+x_{2}^{2}+x_{3}^{2} \leqq 1 ; \quad x_{1}>0, \quad x_{2}>0, \quad x_{3}>0 .
$$

Now it is easy to get a point $\mathbf{u}$ on the surface of the sphere: just project $\mathbf{x}$ radially until it hits the surface; $\mathbf{u}=\rho^{-1} \mathbf{x}$ where $\rho=|\mathbf{x}|$. This is a sample from the uniform distribution on the surface

$$
u_{1}^{2}+u_{2}^{2}+u_{3}^{2}=1 ; \quad u_{1}>0, \quad u_{2}>0, \quad u_{3}>0 .
$$

For our computations, we will need only the squares of the components:

$$
u_{i}^{2}=x_{i}^{2} /\left(x_{1}^{2}+x_{2}^{2}+x_{3}^{2}\right) \quad(i=1,2,3) .
$$

Now we are ready to calculate the distribution function $P(a)$. Suppose we want to calculate $P(a)$ at one or more values $a_{j}$. Let

$$
1<a_{1}<a_{2}<\cdots<a_{n}<T=\left(\lambda_{1} / \lambda_{3}\right)^{1 / 2} \text {. }
$$

Choose $M$, which will be the number of random points $\mathbf{u}$; the error in the Monte Carlo calculation will be of the order $M^{-1 / 2}$.

For convenience, define $a_{n+1}=T$. We will compute sums $s_{1}, s_{2}, \cdots, s_{n+1}$ and then compute

$$
P\left(a_{j}\right)=s_{j} / s_{n+1} \quad(j=1, \cdots, n) .
$$

Initially set all $s_{j}=0$. The sum $s_{j}$ will finally equal the sum of all the random $H(\mathbf{u})$ for which $\mathbf{u}$ lies in $U\left(a_{j}\right)$.

Repeat the following process $M$ times: Compute a random triple $u_{1}^{2}, u_{2}^{2}, u_{3}^{2}$ according to (8.18). Now pick the smallest $a_{m}$ for which $\mathbf{u}$ lies in $U\left(a_{m}\right)$; in other words, let $a_{m}$ be the smallest of the numbers $a_{1}<a_{2}<\cdots<a_{n+1}$ that satisfy the inequality (8.7). (Then $\mathbf{u}$ lies in all the sets $U\left(a_{m}\right), U\left(a_{m+1}\right), \cdots, U\left(a_{n+1}\right)$; but $\mathbf{u}$ does not lie in any of the sets $U\left(a_{j}\right)$ for $j<m$.) For $j=m, \cdots, n+1$, add $H(\mathbf{u})$ to the current value of the sum $s_{j}$. 
After $M$ iterations of the process, compute $P\left(a_{1}\right), \cdots, P\left(a_{n}\right)$ from $(8.20)$. By this program, for each $a=a_{j}$, we compute $P(a)$ by dividing the partial sum (8.15) by the total sum (8.14).

The program uses the mathematical fact that all $\mathbf{u}$ on the unit sphere lie in $U\left(a_{n+1}\right)$. Here is the proof: Since the square-root function is concave,

$$
\left(u_{1}^{2} \lambda_{2} \lambda_{3}+u_{2}^{2} \lambda_{3} \lambda_{1}+u_{3}^{2} \lambda_{1} \lambda_{2}\right)^{1 / 2} \geqq u_{1}^{2}\left(\lambda_{2} \lambda_{3}\right)^{1 / 2}+u_{2}^{2}\left(\lambda_{3} \lambda_{1}\right)^{1 / 2}+u_{3}^{2}\left(\lambda_{1} \lambda_{2}\right)^{1 / 2} .
$$

Set $a=a_{n+1}=\left(\lambda_{1} / \lambda_{3}\right)^{1 / 2}$ and multiply the right-hand side of (8.21) by $a+a^{-1}$; the result is

$$
u_{1}^{2}\left[\left(\lambda_{1} \lambda_{2}\right)^{1 / 2}+\lambda_{1}^{-1 / 2} \lambda_{2}^{1 / 2} \lambda_{3}\right]+u_{2}^{2}\left[\lambda_{3}+\lambda_{1}\right]+u_{3}^{2}\left[\lambda_{1} \lambda_{2}^{1 / 2} \lambda_{3}^{-1 / 2}+\left(\lambda_{1} \lambda_{3}\right)^{1 / 2}\right] .
$$

Since $\lambda_{1} \geqq \lambda_{2} \geqq \lambda_{3}$, this is

$$
\geqq u_{1}^{2}\left[\lambda_{2}+\lambda_{3}\right]+u_{2}^{2}\left[\lambda_{3}+\lambda_{1}\right]+u_{3}^{2}\left[\lambda_{1}+\lambda_{2}\right] .
$$

But that is the left-hand side of (8.7), so $\mathbf{u}$ lies in $U(a)$.

Numerical calculation of $\boldsymbol{p}(\boldsymbol{t})$. If we want a graph of the probability density $p(t)$, we can find it by taking difference-quotients of the distribution function $P(a)$ :

Let $1=a_{0}<a_{1}<\cdots<a_{n+1}=\left(\lambda_{1} / \lambda_{3}\right)^{1 / 2}$. Then the average value of $p(t)$ in the interval $a_{j-1_{i}}<t<a_{j}$ is the difference-quotient

$$
\frac{P\left(a_{j}\right)-P\left(a_{j-1}\right)}{a_{j}-a_{j-1}} .
$$

This is the centered-difference approximation to $p(t)$ at the midpoint $t=$ $\frac{1}{2}\left(a_{j-1}+a_{j}\right)$.

\section{REFERENCES}

[1] W. Feller, An Introduction to Probability Theory and its Applications, vol. I, 3rd ed., John Wiley, New York, 1968.

[2] J. FRANKLIN, Deterministic simulation of random processes, Math. Comp., 17 (1963), pp. 28-59.

[3] J. HAMmERSLEY AND D. HANDSCOMB, Monte Carlo Methods, John Wiley, New York, 1964.

[4] G. Hardy, J. Littlewood ANd G. Pólya, Inequalities, Cambridge University Press, London, 1934.

[5] A. Khintchine, Sur le loi des grands nombres, C. R. Acad. Sci. Paris, 188 (1929), pp. 477-479.

[6] B. KNIGHT, E. WEIBEL AND D. GOMEZ, Effect of size distribution on a principle of counting on sections structures contained in a volume, Proc. First Internat. Congr. Stereol., Vienna, Medical Academy, Vienna, 1963, p. 18.

[7] D. V. LitTle, $A$ third note on recent research in geometrical probability, Advances in Appl. Probability, 6 (1974), pp. 103-130.

[8] P. A. P. MoRAN, A note on recent research in geometrical probability, J. Appl. Probability, 3 (1966), pp. 453-463.

[9] - A second note on recent research in geometrical probability, Advances in Appl. Probability, 1 (1969), pp. 73-89.

[10] E. WEIBEL AND D. GOMEZ, A principle for counting tissue structures on random sections, J. Appl. Physiol., 17 (1962), pp. 343-348.

[11] E. WEIBEL, Stereological principles for morphometry in electron microscopic cytology, Internat. Rev. Cytol., 26 (1969), pp. 235-302. 